\title{
Modelo de intervención psicológica en resiliencia para cuidadores informales de pacientes con Alzheimer ${ }^{*}$
}

\section{Psychological intervention model for informal caregivers of patients with Alzheimer}

\section{Ara Mercedes Cerquera Córdoba** Universidad Pontificia Bolivariana, Colombia \\ Daysy Katherine Pabón Poches Universidad Pontificia Bolivariana, \\ Colombia}

Recibido: 17 de junio de 2014 Revisado: 3 de agosto de 2014 Aceptado: 2 de octubre de 2014

\section{Resumen}

El objetivo del presente estudio es diseñar un modelo de intervención psicológica en resiliencia, con enfoque cognitivo conductual, para cuidadores informales de pacientes con demencia tipo Alzheimer. Se tienen en cuenta las variables depresión, carga, resiliencia, apoyo social y estrategias de afrontamiento, consideradas en la literatura como influyentes en el rol del cuidador. Para realizar el diseño se tiene en cuenta la propuesta metodológica de Mori (2008), y para la evaluación del modelo en su estructura y proceso, la propuesta de evaluación de calidad del servicio de Donabedian (2005). Se concluye que el diseño de programas de intervención para cuidadores informales de pacientes con Alzheimer aparece como una alternativa necesaria para contrarrestar los efectos del cuidado. Este modelo brinda información necesaria para facilitar la réplica en otros contextos, y aporta los elementos requeridos para su implementación.

Palabras clave: modelo, intervención, cuidadores informales, Alzheimer. 


\section{Abstract}

The aim of this study is to design a model of psychological resilience intervention with cognitive behavioral approach for informal caregivers of patients with Alzheimer-type dementia. It takes into account the depression, burden, resilience, social support and coping strategies variables, regarded as influential in the literature on the role of the caregiver. The design takes into account the methodology of Mori (2008) and for the evaluation of both the model structure and process, the quality service evaluation proposal of Donabedian (2005). It is concluded that the design of intervention programs for caregivers of Alzheimer's patients appears as a necessary alternative to counteract the effects of care. This model provides necessary information to facilitate replication in other contexts providing the required elements for implementation.

Keywords: model, intervention, caregivers, Alzheimer.

\section{Introducción}

El Alzheimer (EA) es una enfermedad neurodegenerativa de curso progresivo, muy frecuente en la población anciana (Romano, Nissen, Del Huerto y Parquet, 2007), que afecta al adulto mayor (AM) y a sus familiares. Generalmente uno de los integrantes se encarga del cuidado de aquel, y es denominado cuidador informal. Esta persona provee ayuda no remunerada, usualmente en el contexto doméstico; invierte grandes cantidades de tiempo y energía durante meses o años; tiene un elevado compromiso con su labor; se caracteriza por el afecto y la atención sin límite de horarios; y asiste a la persona dependiente en actividades básicas e instrumentales (Aparicio, Díaz, Cuéllar, Fernández y De Tena, 2008; Lavretsky, 2005).

Desempeñar este rol incluye experimentar en algunas ocasiones depresión (Cerquera, Pabón y Uribe, 2012; Lladó, Antón-Aguirre, Villar, Rami y Molinuevo, 2008), ansiedad (Ferrera et al., 2008; Paleo y Rodríguez, 2005), sentimiento de culpa (Sanders, Ott, Kelber y Noonan, 2008), carga (Cerquera, Granados y Buitrago, 2012; McCurry, Logdson, Terri y Vitielo, 2007), altos niveles de estrés (Paleo y Rodríguez, 2005), bajos niveles de satisfacción con la vida, y problemas laborales y legales (Arango-Lasprilla, Rogers y FernándezGuinea, 2003).

Ante los hallazgos mencionados con anterioridad, se han implementado formas de intervenir con los cuidadores, donde se incluyen los programas de respiro, programas de autoayuda, psico-educativos y psicoterapéuticos (Aparicio et al., 2008). Dentro de aquellas se destacan las intervenciones psicológicas como las más eficaces, pues sus efectos van más allá de la información o ayuda material e instrumental, interfieren en las características personales del cuidador y promueven cambios más duraderos en el tiempo (Zabalegui et al., 2008; Orozco-Gómez, Eustache-Rodríguez y Grosso-Torres, 2012; Losada, et. al., 2005).

Para este caso, la intervención psicológica se enfoca en la resiliencia, descrita como una capacidad universal, que puede ser promovida a lo largo de los ciclos de vida, y está vinculada con el desarrollo y el crecimiento humano (Gómez, 2010). Cuando una persona se enfrenta a una situación adversa, de riesgo o traumática, la enfrenta de modo que pueda normalizar su vida sacando provecho de la experiencia, mas no desarrollando patologías (Carretero, 2010). Así, se asume una intervención con perspectiva salutogénica, donde la investigación se centra en las cualidades y emociones positivas que posee el individuo (Vera, 2006).

Aunque la resiliencia presenta varias conceptualizaciones, para el diseño de este modelo se toma como base la definición de Wagnild y Young (1993), quienes la describen como una característica de personalidad positiva que permite la adaptación del individuo ante situaciones amenazantes. Según Salgado (2005), la resiliencia inclu- 
ye dos factores: 1) competencia personal, la cual indica auto-confianza, independencia, decisión, invencibilidad, poderío, ingenio y perseverancia; y 2 ) aceptación de uno mismo y de la vida, la cual representa adaptabilidad, balance, flexibilidad y una perspectiva de vida estable; la persona refleja aceptación por la vida y un sentimiento de paz, a pesar de la adversidad.

El modelo pretende abordar la resiliencia como centro de la intervención, pues es una característica que mantiene el funcionamiento adaptativo de las funciones físicas y psicológicas en situaciones adversas (Vera, Carbonela y Vecina, 2012). En el caso de los cuidadores, la resiliencia se asocia a un mejor estado emocional y físico de aquellos, así como a variables propias del cuidador como percepción y afrontamiento, características de la personalidad, más que a variables situacionales (Fernández-Lansac, Crespo, Cáceres y Rodríguez, 2012). Así, cuidadores más resilientes benefician en mayor medida a los adultos mayores cuidados en contexto familiar (Oliveira, Ferreira y Ferreira, 2012).

Además, se ha encontrado que esta característica se evidencia en personas que, aun habiendo vivido una situación traumática, han conseguido encajarla y seguir viviendo incluso en un nivel superior, como si la situación traumática vivida y asumida hubiera desarrollado en ellas recursos latentes e insospechados (Colom, Domingo, Gracia y Medina, 2012), situación que se desea resaltar en la forma en que los cuidadores asumen el cuidado.

Para poder intervenir sobre la resiliencia, el modelo se acoge al enfoque cognitivo conductual, un enfoque donde el terapeuta ayuda a los cuidadores a desarrollar habilidades de solución de problemas, a organizar el tiempo y a realizar actividades gratificantes en la medida de lo posible (López, 2005). Este tipo de enfoque busca que el cuidador tenga la mejor adaptación posible a la situación de carga con la que se enfrenta, por medio de la modificación de conductas, pensamientos y emociones desadaptativas (López, 2005). Esto se evidencia en los hallazgos de Losada et al. (2005), quienes encontraron que por medio de la intervención cognitivo-conductual se logró un cambio significativo en la evaluación pre-pos test, a diferencia de un programa psicoeducativo y el grupo control, resultados que además se mantuvieron en el seguimiento de la intervención tres meses después.

Asimismo, Zabalegui et al. (2008) afirman que las intervenciones con participación activa de los cuidadores, y basadas en terapia cognitivo-conductual, son más eficaces que aquellas cuyo contenido se centra exclusivamente en la adquisición de conocimientos. Por su parte, Orozco-Gómez et al. (2012), quienes desarrollaron un programa de intervención cognitivo conductual, resaltaron la importancia de intervenir terapéuticamente sobre las variables conductuales y cognoscitivas que interactúan en el proceso de formación, apoyo y manejo de la salud de los cuidadores familiares.

En este sentido, este modelo de intervención se orienta bajo el modelo cognitivo-conductual, con un enfoque multimodal donde se abordan los pensamientos, emociones y comportamientos, centrándose en los aspectos positivos que puede incluir el hecho de cuidar a un familiar con Alzheimer. Igualmente se incluye el ejercicio físico en cuanto a respiración, relajación e intervención terapéutica musical, como parte de las estrategias de intervención.

Para la evaluación de este modelo, se toma el modelo de calidad del servicio de Donabedian (2001), el cual incluye una evaluación de estructura, que refiere a todos los recursos materiales, organizacionales, humanos y financieros disponibles en los sitios en los que se proporciona atención (García, Orrego y Blasco, 2010), y una evaluación de proceso, la cual refiere a lo que los prestadores del servicio y proveedores son capaces de hacer por los pacientes, la interrelación con las actividades de apoyo diagnosticadas, y las actitudes, habilidades, destrezas y técnicas con que se llevan a cabo (Donabediam, 2005). Esto, con la finalidad de controlar y hacer visibles los efectos del modelo de intervención psicológico.

\section{Método}

Es un estudio mixto, que combina el análisis cuantitativo y cualitativo para validar el modelo de intervención, a partir del uso de dos tipos de eva- 
luación basados en el modelo de calidad de atención médica de Donabedian (2005), evaluación de estructura y de proceso.

\section{Participantes}

El modelo de intervención psicológico para cuidadores informales cuenta con la participación de dos psicólogos pertenecientes a la línea de investigación Calidad de Vida en la Tercera Edad, de la Universidad Pontificia Bolivariana, quienes formulan y diseñan el modelo, y dos psicoterapeutas con experiencia clínica, que cumplieron el papel de jueces externos.

\section{Instrumentos}

Para el diseño del protocolo se utilizó la propuesta metodológica adaptada de Mori (2008), quien plantea ocho fases para el diseño, ejecución y evaluación de programas. La primera fase es el diagnóstico de la comunidad; la segunda fase se refiere a las características del grupo; la tercera fase es la evaluación de necesidades; la cuarta fase es el diseño y planificación de la intervención; la quinta fase propone una evaluación de los contenidos por desarrollar en la intervención; la sexta fase es la ejecución y la implementación del programa; la séptima fase es la evaluación final; y la octava fase propone una diseminación de los resultados.

Para la formulación del modelo se construyó un protocolo de intervención basado en el enfoque cognitivo conductual. Para la evaluación del modelo se tuvo en cuenta el modelo de evaluación de calidad del servicio, adaptado de Donabedian (2001). Así, se diseñaron dos protocolos: un protocolo de evaluación de estructura, conformado por diez ítems, y un protocolo de evaluación de proceso, que incluye una evaluación por parte del cuidador, compuesta por ocho ítems; una evaluación final del programa, con un cuestionario de dieciséis reactivos; y una evaluación por parte del terapeuta, constituida por dos ítems.

\section{Procedimiento}

Fase I. Diseño del protocolo. Se construye el modelo de intervención en resiliencia, basado en la propuesta metodológica adaptada de Mori (2008); posteriormente se somete a un proceso de validación por jueces expertos en sesiones de discusión.

Fase II. Formulación del protocolo de intervención psicológica en resiliencia. Se tiene en cuenta el modelo cognitivo conductual, que consta de diez sesiones, con una duración aproximada de noventa minutos cada una; va dirigido a cuidadores informales de pacientes con demencia tipo Alzheimer, de 18 años en adelante -sin límite de edad-, de cualquier nacionalidad y estrato socioeconómico. Se trabaja de forma grupal, con máximo veinticinco personas.

Fase III. Formulación de los protocolos de evaluación de estructura y de proceso. Están guiados por el modelo de calidad de atención médica de Donabedian (2005), el cual insiste en la necesidad de evaluar de forma integral cualquier servicio de salud.

\section{Resultados}

Para diseñar el modelo de intervención psicológi$\mathrm{ca}$ en resiliencia se siguieron las siguientes pautas:

Tabla 1

Diseño del modelo de intervención en resiliencia

\begin{tabular}{cl}
\hline Fase & Descripción \\
\hline 1 & $\begin{array}{l}\text { Evaluación de las necesidades de los cuida- } \\
\text { dores. }\end{array}$ \\
\hline 2 & Caracterización de los cuidadores. \\
\hline 3 & $\begin{array}{l}\text { Diagnóstico de los cuidadores y evaluación } \\
\text { preliminar (aplicación pre-test). }\end{array}$ \\
\hline 4 & Diseño del modelo de intervención. \\
\hline 5 & $\begin{array}{l}\text { Evaluación inicial del contenido del modelo } \\
\text { (revisión de contenidos). }\end{array}$ \\
\hline 6 & $\begin{array}{l}\text { Ejecución del modelo de intervención para } \\
\text { cuidadores. }\end{array}$ \\
\hline 7 & Evaluación de estructura y de proceso. \\
\hline 8 & Replicabilidad del modelo. \\
\hline
\end{tabular}

Fuente: elaboración propia. 
La tabla 1 muestra la forma en que se adaptó la propuesta metodológica de Mori (2008) al contenido del modelo de intervención en resiliencia. La aplicación de este modelo incluye un pretest para verificar el estado inicial del cuidador, evaluando las variables mencionadas con anteriori- dad; una aplicación postest, para identificar los cambios efectuados por el modelo en el cuidador; y un seguimiento durante tres meses después de terminado el programa de intervención, para determinar el impacto de esta.

Tabla 2

Estructura general del modelo de intervención

\begin{tabular}{llll}
\hline Nombre sesión & Objetivo & Contenidos & Técnicas \\
\hline \multirow{2}{*}{ Presentación } & $\begin{array}{ll}\text { Establecer contrato terapéu- } \\
\text { tico con los cuidadores. }\end{array}$ & $\begin{array}{l}\text { Descripción del modelo, ob- } \\
\text { jetivo y justificación. Presen- } \\
\text { tación de terapeutas y cuida- } \\
\text { dores. }\end{array}$ \\
\hline
\end{tabular}

$\begin{array}{ll} & \text { Mejorar la imagen que se tie- } \\ \text { Este soy yo } & \text { ne de sí mismo, reconociendo } \\ \text { emociones y facilitando la au- } \\ \text { toconfianza. }\end{array}$

Mejorar la imagen que se tieemociones y facilitando la autoconfianza.
Autoimagen, reconocimiento de emociones, autoconfianza.
Auto-reconocimiento, introspección, respiración, relajación.
Aceptándome

Reconocer por medio de las experiencias positivas las características salutogénicas para aceptarse a sí mismo.
Experiencias positivas, sesión del futuro, autovaloración, aceptación de sí mismo y de la vida.
Plenaria, Respiración, relajación, reconocimiento, imaginería.
Identificar el tipo de apoyo social que tiene el cuidador para potenciar el balance personal y reconocer la satisfacApoyo social ción en el acto de agradecer. Reconocer en la satisfacción personal una característica importante de la aceptación de sí mismo y de la vida.
Apoyo social, satisfacción personal, balance personal.
Respiración, relajación, modelamiento, solución de problemas, interpretación.
Reconocer las cualidades que

Así me ven otros ven en uno, para fortalecer la confianza en sí mismo río. e incrementar el poderío.
Confianza en sí mismo, poderío.

Reconocer la perseverancia y la decisión como competencias personales desarrolladas.

Queriéndolo hace y lo logré

El objetivo de la sesión es

Lo que sería capaz de hacer

identificar la decisión como una característica importante de la competencia personal.

Reconociendo mis derechos y aprendiendo a ayudar
Analizar que al reconocer los derechos como cuidador lograría potenciar la característica de una vida estable.
Competencias personales, perseverancia y decisión.

Competencia personal, capacidad de decisión.

Derechos de los cuidadores.
Narrativa, respiración y relajación, interpretación, modelamiento.
Respiración, relajación, intervención terapéutica y musical, solución de problemas, plenaria. Respiración, relajación, imaginería, intervención terapéutica musical, interpretación, plenaria.

Relajación, respiración, intervención terapéutica musical, imaginería, plenaria. 


\begin{tabular}{|c|c|c|c|}
\hline Nombre sesión & Objetivo & Contenidos & Técnicas \\
\hline $\begin{array}{l}\text { Mis poderes moti- } \\
\text { vacionales }\end{array}$ & $\begin{array}{l}\text { Inhibir acciones, sentimien- } \\
\text { tos o creencias indeseables } \\
\text { con el fin de que el cuida- } \\
\text { dor visualice la aparición de } \\
\text { logros y objetivos que lleven } \\
\text { al individuo a desarrollar las } \\
\text { competencias personales de } \\
\text { la resiliencia. }\end{array}$ & Invencibilidad e ingenio. & $\begin{array}{l}\text { Relajación, respiración, ima- } \\
\text { ginería, intervención tera- } \\
\text { péutica musical, confronta- } \\
\text { ción, plenaria. }\end{array}$ \\
\hline Cierre & $\begin{array}{l}\text { Evaluar el modelo de inter- } \\
\text { vención. }\end{array}$ & $\begin{array}{l}\text { Imágenes del registro de la } \\
\text { ejecución. }\end{array}$ & Ninguna. \\
\hline
\end{tabular}

Fuente: elaboración propia.

En la tabla 2 se encuentra la estructura del modelo, el cual consta de diez sesiones; ocho corresponden a la ejecución del modelo de intervención, y dos, la inicial y la final, a la presentación y al cierre. Se sugiere que el modelo sea aplicado una vez por semana, con una duración de noventa minutos por sesión. Para la evaluación del programa de intervención, se tienen en cuenta criterios de estructura y de proceso, basados en el modelo de calidad de atención médica de Donabedian (2005).

Tabla 3

Rúbrica de evaluación de estructura PIRCA

Evaluación de estructura

Puntaje óptimo (\%) Puntaje obtenido

1. Conocimiento del área de responsabilidad del centro

\begin{tabular}{ll}
\hline Se dispone del croquis del instituto & 33.3 \\
\hline Se dispone de datos personales de los cuidadores & 33.3 \\
\hline Se dispone de datos actualizados de los cuidadores & 33.3 \\
\hline Total & 100 \\
\hline 2. Información acerca de las necesidades de la población & 33,3 \\
\hline Se conocen datos de participantes & 33,3 \\
\hline Se tiene un diagnóstico de los cuidadores & 33,3 \\
\hline Existe un perfil de las necesidades del cuidador & 100 \\
\hline Total & \\
\hline 3. Características del personal de la entidad & 20 \\
\hline La terapeuta del proyecto tiene experiencia en investigación & 20 \\
\hline La terapeuta del proyecto tiene experiencia en el área clínica & 20 \\
\hline La coterapeuta del proyecto tiene experiencia en investigación & 20 \\
\hline La coterapeuta del proyecto tiene experiencia en el área clínica & 20 \\
\hline Los colaboradores del semillero han sido capacitados para su función & 100 \\
\hline Total & \\
\hline
\end{tabular}


Evaluación de estructura

Puntaje óptimo (\%) Puntaje obtenido

\begin{tabular}{lc}
\hline 4. Disponibilidad de equipamiento para las actividades & \\
\hline Video beam & 25 \\
\hline Papelería & 25 \\
\hline Material de escritorio & 25 \\
\hline Reproductor de música & 25 \\
\hline Total & 100
\end{tabular}

5. Disponibilidad de insumos

\begin{tabular}{lc}
\hline Lapiceros & 50 \\
\hline Fotocopias & 50 \\
\hline Total & 100
\end{tabular}

6. Disponibilidad de formularios de registro de actividades

\begin{tabular}{lc} 
Asistencia por sesión & 33,3 \\
\hline Pruebas psicológicas del protocolo de aplicación & 33,3 \\
\hline Entrevista semiestructurada & 33,3 \\
\hline Total & 100
\end{tabular}

7. Disponibilidad de normas escritas para las actividades

\begin{tabular}{ll} 
Manual del programa de intervención & 50 \\
\hline Instrucciones por sesión & 50 \\
\hline Total & 100 \\
\hline
\end{tabular}

8. Planta física

Recepción

Dispone de un ambiente amplio con disponibilidad de asientos $\quad 12,5$

Mantiene aceptablemente limpio el lugar $\quad 12,5$

Dispone de luz adecuada durante la estancia del paciente $\quad 12,5$

$\begin{array}{ll}\text { Dispone de baños para el público } & 12,5\end{array}$

Salón de sesiones

Es un espacio amplio con disponibilidad de asientos para los asistentes $\quad 12,5$

Mantiene aceptablemente limpio el lugar 12,5

Dispone de luz adecuada durante la estancia del paciente $\quad 12,5$

Dispone de baños para el público 12,5

$\begin{array}{ll}\text { Total } & 100\end{array}$

9. Trabajo comunitario

Se realizan visitas domiciliarias programadas a los cuidadores 50

Se realizan llamadas recordatorias a los cuidadores $\quad 50$

$\begin{array}{ll}\text { Total } & 100\end{array}$

\begin{tabular}{lc}
\hline 10. Recursos financieros & \\
\hline El proyecto cuenta con presupuesto para la ejecución total & 50 \\
\hline Se cuenta con presupuesto para todas las necesidades del proyecto & 50 \\
\hline Total & 100
\end{tabular}

Fuente: elaboración propia. 
En la tabla 3 se encuentra la evaluación de estructura, tomando como base la guía de evaluación adaptada de Moreno (2001). La evaluación de estructura requiere información disponible por parte de directivos del centro y miembros o funcionarios de aquel, para mejorar su funcionamiento. La rúbrica está dividida en diez aspectos, cada uno de los cuales se subdivide en ítems, según el modelo de intervención. Cada aspecto equivale a un $100 \%$, el cual es dividido proporcionalmente según el número de ítems, dando un puntaje esperado.

Con los datos obtenidos en la rúbrica, se elabora la tabla 4, y se obtiene un consolidado por medio de la sumatoria de los puntos de cada aspecto evaluado. Luego se compara esta información con el puntaje obtenido, el cual es la calificación que asigna el evaluador, teniendo en cuenta la presencia o ausencia del ítem, donde la presencia equivale al puntaje óptimo, y la ausencia, a cero. Después se calcula el porcentaje logrado.

En la tabla 4 se refleja el consolidado de la rúbrica, de la cual se tiene en cuenta el porcentaje obtenido para señalar la calificación. Un puntaje de $80 \%$ en adelante significa bueno; $60 \%-79 \%$, regular; y menos de $60 \%$, malo.

Para la evaluación del proceso se crean tres guías de evaluación: evaluación por parte del cuidador, evaluación por parte del terapeuta y evaluación final del modelo.

Tabla 4

Consolidado de puntuaciones de evaluación de estructura PIRCA

\begin{tabular}{|c|c|c|c|}
\hline Rubros & $\begin{array}{l}\text { Puntaje } \\
\text { óptimo }\end{array}$ & $\begin{array}{l}\text { Puntaje } \\
\text { obtenido }\end{array}$ & $\begin{array}{c}\text { Porcentaje } \\
\text { Logrado }\end{array}$ \\
\hline 1. Conocimiento del área de responsabilidad e influencia del centro & 100 & & \\
\hline 2. Información acerca de las necesidades de la población & 100 & & \\
\hline 3. Características del personal de la entidad & 100 & & \\
\hline 4. Disponibilidad de equipamiento para las actividades & 100 & & \\
\hline 5. Disponibilidad de insumos & 100 & & \\
\hline 6.Disponibilidad de formularios de registro de actividades & 100 & & \\
\hline 7. Disponibilidad de normas escritas para las actividades & 100 & & \\
\hline 8. Planta física & 100 & & \\
\hline 9. Trabajo comunitario & 100 & & \\
\hline 10. Recursos financieros & 100 & & \\
\hline Total & 1000 & & \\
\hline
\end{tabular}

Fuente: Moreno (2001).

Tabla 5

Evaluación por parte del cuidador

\begin{tabular}{lllllll}
\hline 1 & Satisfacción con el contenido teórico & 1 & 2 & 3 & 4 & 5 \\
\hline 2 & Satisfacción con el contenido práctico & 1 & 2 & 3 & 4 & 5 \\
\hline 3 & Satisfacción con los terapeutas & 1 & 2 & 3 & 4 & 5 \\
\hline 4 & Claridad de la información transmitida & 1 & 2 & 3 & 4 & 5 \\
\hline 5 & Adecuación de los ejercicios & 1 & 2 & 3 & 4 & 5 \\
\hline 6 & Adecuación de los materiales & 1 & 2 & 3 & 4 & 5 \\
\hline 7 & Responde a las expectativas & 1 & 2 & 3 & 4 & 5 \\
\hline 8 & Utilidad de lo aprendido & 1 & 2 & 3 & 4 & 5 \\
\hline
\end{tabular}

Fuente: elaboración propia. 
En la tabla 5 se observan los componentes evaluados por el cuidador. Teniendo en cuenta su apreciación, aquel evalúa de 1 a 5 , siendo 1 la categoría de poca satisfacción, y 5 , la máxima satisfacción. Debe marcar el número en la casilla que considere que más se ajusta a su apreciación.
En la tabla 6 se observan los criterios que evalúa el terapeuta en relación con el cumplimiento y la calidad de las tareas asignadas al cuidador.

En la tabla 7 se reflejan los aspectos evaluados por el cuidador al final de la intervención.

Tabla 6

Evaluación por parte del terapeuta

\section{Criterios para la cuantificación del número de tareas para la casa realizadas}

0 Las tareas para la casa y los ejercicios de la sesión no han sido realizados.

1 Se ha realizado menos de la mitad de las tareas y ejercicios.

2 Se ha realizado la mitad de las tareas y ejercicios.

3 Se ha realizado más de la mitad de las tareas y ejercicios.

4 Se ha realizado la totalidad de las tareas y ejercicios.

Criterios para la cuantificación de la calidad de la tarea: Registro

0 El cuidador no ha hecho la tarea.

1 El cuidador ha tenido dificultades para realizar la tarea (buscar el momento adecuado).

2 Las habilidades han sido implementadas, pero de una manera equivocada.

3 Las habilidades se han implementado, pero se deben hacer ajustes (no se han hecho todos los días).

$4 \quad$ Las habilidades se han implementado correctamente

5 Las habilidades se han implementado correctamente, y el cuidador realiza observaciones o cambios.

6 Las habilidades se han implementado correctamente y se han generalizado a otras situaciones o problemas.

Fuente: elaboración propia.

Tabla 7

Evaluación final del modelo de intervención

\begin{tabular}{|c|c|c|c|}
\hline \multirow{4}{*}{$\begin{array}{l}\text { 1. ¿Cómo calificaría la } \\
\text { calidad del servicio que ha } \\
\text { recibido? }\end{array}$} & Excelente & \multirow{4}{*}{$\begin{array}{l}\text { 9. ¿Los terapeutas contaban con } \\
\text { el profesionalismo y habilidades } \\
\text { adecuadas para llevar a cabo el } \\
\text { programa de intervención? }\end{array}$} & Mucho \\
\hline & Buena & & Bastante \\
\hline & Regular & & Poco \\
\hline & Mala & & Nada \\
\hline \multirow{4}{*}{$\begin{array}{l}\text { 2. ¿En qué medida nuestro } \\
\text { programa ha satisfecho sus } \\
\text { necesidades? }\end{array}$} & Totalmente & \multirow{4}{*}{$\begin{array}{l}\text { 10. ¿Recibió conocimientos úti- } \\
\text { les para afrontar la labor de } \\
\text { cuidador? }\end{array}$} & Mucho \\
\hline & En general & & Bastante \\
\hline & Parcialmente & & Poco \\
\hline & Ninguna & & Nada \\
\hline \multirow{4}{*}{$\begin{array}{l}\text { 3. Si un amigo necesitara } \\
\text { una ayuda similar, ¿le } \\
\text { recomendaría nuestro } \\
\text { programa? } \\
\text { a. No }\end{array}$} & No & \multirow{4}{*}{$\begin{array}{l}\text { 11. ¿Con qué frecuencia utiliza los } \\
\text { ejercicios enseñados en las } \\
\text { sesiones del programa? }\end{array}$} & Siempre \\
\hline & Probablemente no & & Casi siempre \\
\hline & Probablemente sí & & Algunas veces \\
\hline & sí & & Nunca \\
\hline
\end{tabular}




\begin{tabular}{|c|c|c|c|}
\hline \multirow{4}{*}{$\begin{array}{l}\text { 4. ¿En qué medida está } \\
\text { satisfecho con el tipo de } \\
\text { ayuda recibida? }\end{array}$} & Mucho & \multirow{4}{*}{$\begin{array}{l}\text { 12. La percepción que usted tiene } \\
\text { de los terapeutas es: }\end{array}$} & Excelente \\
\hline & Bastante & & Buena \\
\hline & Poco & & Regular \\
\hline & Nada & & Mala \\
\hline \multirow{4}{*}{$\begin{array}{l}\text { 5. ¿Le han ayudado } \\
\text { los servicios que ha } \\
\text { recibido a hacer frente } \\
\text { más eficazmente a sus } \\
\text { problemas? }\end{array}$} & Mucho & \multirow{4}{*}{$\begin{array}{l}\text { 13. ¿Usted realizó las tareas del } \\
\text { programa de intervención para } \\
\text { trabajar en casa? }\end{array}$} & Siempre \\
\hline & Bastante & & Casi siempre \\
\hline & Poco & & Algunas veces \\
\hline & $\mathrm{Nada}$ & & Nunca \\
\hline \multirow{4}{*}{$\begin{array}{l}\text { 6. ¿La información brindada } \\
\text { por los terapeutas fue } \\
\text { clara? }\end{array}$} & Mucho & \multirow{4}{*}{$\begin{array}{l}\text { 14. ¿Ha podido utilizar los ejerci- } \\
\text { cios y la información recibida } \\
\text { en el programa de intervención } \\
\text { para otras áreas de su vida dife- } \\
\text { rentes a la labor de cuidador? }\end{array}$} & Mucho \\
\hline & Bastante & & Bastante \\
\hline & Poco & & Poco \\
\hline & $\mathrm{Nada}$ & & Nada \\
\hline \multirow{4}{*}{$\begin{array}{l}\text { 7. ¿Siente que comprendió } \\
\text { adecuadamente la } \\
\text { información brindada por } \\
\text { los terapeutas? }\end{array}$} & Mucho & \multirow{4}{*}{$\begin{array}{l}\text { 15. En conjunto, ¿en qué medida } \\
\text { está satisfecho con el servicio } \\
\text { recibido? }\end{array}$} & Mucho \\
\hline & Bastante & & Bastante \\
\hline & Poco & & Poco \\
\hline & Nada & & Nada \\
\hline \multirow{4}{*}{$\begin{array}{l}\text { 8. ¿Los materiales, contenidos } \\
\text { y ejercicios ejecutados } \\
\text { en el programa fueron } \\
\text { adecuados? }\end{array}$} & Mucho & \multirow{4}{*}{$\begin{array}{l}\text { 16. Si tuviera que buscar ayuda } \\
\text { otra vez, ¿volvería a nuestro } \\
\text { programa? }\end{array}$} & No \\
\hline & Bastante & & Probablemente no \\
\hline & Poco & & Probablemente sí \\
\hline & Nada & & Sí \\
\hline
\end{tabular}

Fuente: elaboración propia.

\section{Discusión y conclusiones}

El diseño de programas de intervención para cuidadores informales de pacientes con Alzheimer aparece como una alternativa necesaria para contrarrestar los efectos del cuidado, la cual contribuye a la disminución del malestar psicológico y emocional del cuidador (Martínez, Villalba y García, 2001).

Este modelo brinda información necesaria para facilitar la réplica en otros contextos, aportando los elementos requeridos para su implementación, como son el diseño, la metodología, la evaluación de estructura y el proceso. Según la Unicef (2008); Brodaty, Green y Koschera (2003), para la elaboración de una intervención es necesario tener una estructura, un modelo o un diseño guía, para dar crédito a la validez de lo que se está creando. El modelo de intervención se apoyó en la propuesta de Mori (2008), con la intención de tener una base sólida desde la elaboración, además de los cimientos teóricos y empíricos, ya que las intervenciones con cuidadores requieren una metodología específica, acorde con las características de aquellos (Losada-Baltar y Montorio-Cerato, 2005).

Este modelo plantea una intervención psicológica, apoyada en Espín (2008); Losada et al. (2005); Zabalegui et al. (2008) y Orozco-Gómez et al. (2012), quienes afirman que las intervenciones psicoeducativas o informativas no han sido suficientes para mejorar la calidad de vida del cuidador, mientras que la intervención psicológica se presenta como un medio más adecuado para intervenirlo. Ya se ha evidenciado que las intervenciones con participación activa de los cuidadores, y basadas en terapia cognitivo-conductual, son más eficaces que aquellas cuyo contenido se centra exclusivamente en la adquisición de conocimientos (Zabalegui et al., 2008). 
Se recomienda que junto con la aplicación del modelo, se vincule otro tipo de apoyo psicosocial que permita mayores efectos. Se sabe que las características de los cuidadores no permiten disponibilidad de tiempo, desánimo, desinterés o retiro de los programas de intervención; en este sentido, contar con programas de respiro o de apoyo formal puede dar más espacio, tiempo y disposición a los cuidadores, para asistir y participar en las intervenciones psicológicas (Losada-Baltar y Montorio-Cerato, 2005).

\section{Referencias}

Arango-Laspriella, J., Rogers, H. \& FernándezGuinea, S. (2003). La familia y el cuidador del paciente con demencia y sus recursos. En J. Arango-Laspriella., S. Fernández-Guinea y A. Ardila (eds.), Las demencias. Aspectos clínicos, neuropsicológicos y tratamiento. México, D. F.: Manuel Moderno.

Aparicio, M., Díaz, J., Cuéllar, B., Fernández, M. \& De Tena, A. (2008). La salud física y psicológica de las personas cuidadoras. Comparación entre cuidadores formales e informales (tesis inédita de doctorado). Universidad Complutense de Madrid, Madrid, España.

Brodaty, H., Green, A., \& Koschera, A. (2003). Metaanalysis of psychosocial interventions for caregivers of people with dementia. Journal of the American Geriatrics Society, 51, 657-664.

Carretero, R. (2010). Resiliencia. Una revisión positiva para la prevención e intervención desde los servicios sociales. Nómadas. Revista Crítica de Ciencias Sociales y Jurídicas, 27(3), 1-13.

Cerquera, A., Granados, F. \& Buitrago, A. (2012). Sobrecarga en cuidadores de pacientes con demencia tipo Alzheimer. Psichologya. Avances de la Disciplina, 6(1), 35-45.

Cerquera, A., Pabón, D. \& Uribe, D. (2012). Nivel de depresión experimentada por una muestra de cuidadores informales de pacientes con demencia tipo Alzheimer. Psicología desde el Caribe, 29(2), 360-384.
Colom, C., Domingo, N., Gracia, H. \& Medina, V. (2012). Psicología positiva, asesoramiento psicológico. Recuperado el 31 de julio de 2013 de http://www.sc.ehu.es/ptwpefej/docencia/asesoramiento\%20psicologico/trabajos/2012/19_psicologia_positiva.pdf

Donabedian, A. (2001). Reflexiones sobre calidad asistencial. Revista Calidad Asistencial, 16, 79-143.

Donabedian, A. (2005). Evaluating the quality of medical care. The Milbank Memorial Fund Quarterly, 83(4), 691-729.

Espín, A. (2008). Caracterización psicosocial de cuidadores informales de adultos mayores con demencia. Revista Cubana de Salud Pública, 34(3), 1-13.

Fernández-Lansac, V., Crespo, M., Cáceres, R., \& Rodríguez, M. (2012). Resiliencia en cuidadores de personas con demencia: Estudio preliminar. Revista Española de Geriatría y Gerontología, 43(3), 102-109.

Ferrera, M., Languiano, E., Di Brango, T., De Vito, E., Di Cioccio, L., \& Bauco, C. (2008). Prevalence of stress, anxiety and depression with Alzheimer Caregivers. Health Qual. Life Outcomes, 6(93). Dol: 10.1186/1477-7525-6-93.

García, J., Orrego, C. Blasco, J. (2010). Evaluación de la implementación. Implementación de guías de práctica clínica en el sistema nacional de salud. Manual metodológico. Recuperado el 24 de enero de 2014 de http://portal.guiasalud.es/emanuales/implementacion/ apartado05/evaluacion.html

Gómez, B. (2010). Resiliencia individual y familiar. Recuperado el 12 de febrero de 2013 de http://www.avntfevntf.com/imagenes/ biblioteca/G\%C3\%B3mez,\%20B.\%20Trab.\%20 3\%C2\%BA\%20BI\%2009-10.pdf

Lavretsky, H. (2005). Stress and depression in informal family caregivers of patients with Alzheimer's disease. Aging Health, 1(1), 117-133.

López, J. (2005). Entrenamiento del manejo del estrés en cuidadores de familiares mayores 
dependientes. Desarrollo y evaluación de la eficacia de un programa (tesis inédita de doctorado). Universidad Complutense de Madrid, Madrid, España.

Losada-Baltar, A. \& Montorio-Cerato. (2005). Pasado, presente y futuro de las intervenciones psicoeducativas para cuidadores familiares de personas mayores dependientes. Revista Especialista de Geriatría y Gerontología, 40(3), 30-9.

Losada, A., Montorio, I., Cerato, I., Fernández, M. \& Márquez, M. (2005). Estudio e intervención sobre el malestar psicológico de los cuidadores de personas con demencia. El papel de los pensamientos disfuncionales. Madrid: IMSERSO.

Lladó, A., Atón-Aguirre, S., Villar, A., Rami, L. \& Molinuevo, J. (2008). Impacto psicológico del diagnóstico de la enfermedad de Alzheimer. Neurología, 23(25), 294-298.

Martínez, M., Villalba, C. García, M. (2001). Programas de respiro para cuidadores familiares. Intervención Psicosocial, 10(81), 7-22.

McCurry, S., Logdson, R., Teri, L. \& Vitielo, M. (2007). Sleep disturbances in caregivers of person with dementia: Contributing factors and treatment implications. Sleep Medicine Reviews, 11(2), 143-153.

Moreno, E., Crivelli, V., Kesteman, N., Malé, M., Passarell, N., Schapira, E. \& Viñas, M. (2001). Calidad de la atención primaria. Aporte metodológico para su evaluación. Recuperado de http://portales.puj.edu.co/ftpcentroescritura/Recursos/Normasapa.pdf

Mori, M. (2008). Una propuesta metodológica para la intervención comunitaria. Liberabit, 14, 81-90.

Oliveira, C., Ferreira, A. \& Ferrria, J. (2012). Perfil de cuidadores de idosos com doença de Alzheimer associado à resiliência. Texto \& Contexto Enfermagem, 21(1), 150-157.

Orozco-Gómez, A., Eustache-Rodríguez, V. \& Grosso-Torres, L. (2012). Programa de inter- vención cognoscitivo conductual en la calidad de sueño de cuidadores familiares. Revista Colombiana de Enfermería, 7(7), 75-85.

Paleo, N. \& Rodríguez, N. (2005). ¿Por qué cuidar a los cuidadores de pacientes con enfermedad de Alzheimer? Revista Electrónica de Geriatría, 7. Recuperado el 1 de agosto de 2011 de www.geriatrinet.com/introx.htlm

Romano, M., Nissen, M., Del Huerto, N. \& Parquet, C. (2007). Enfermedad de Alzheimer. Revista de Posgrado de la Vía Cátedra de Medicina, 75, 9-12.

Salgado, A. (2005). Métodos e instrumentos para medir la resiliencia: Una alternativa Peruana. Liberabit, 11, 42-48.

Sanders, S., Ott, C., Kelber, S., \& Noonan, P. (2008). The experience of high levels of grief in caregivers of persons with Alzheimer's disease and related demential. Deathstud, 32(6), 495-523.

UNICEF (2006). Elaborando proyectos de comunicación para el desarrollo. Buenos Aires: Silvina Chemen.

Vera, B. (2006). Psicología positiva. Una nueva forma de entender la psicología. Papeles del Psicólogo, 27(1), 3-8.

Vera, B., Carbelo, B. \& Vecina, M. (2012). La experiencia traumática desde la psicología positiva. Resiliencia y crecimiento postraumático. Papeles del Psicólogo, 27(1), 40-49.

Wagnild, G. M. \& Young, H. M. (1993). Development and psychometric evaluation of the resilience scale. Journal of Nursing Measurement, 1, 165-178.

Zabalegui, A., Monserrat, D., Cabrera, E., Fernández-Puebla, A., Bardallo, D., Rodríguez, E., ... Remon, A. (2008). Eficacia de las intervenciones dirigidas a cuidadores principales de personas dependientes mayores de 65 años. Una revisión sistémica. Revista Española de Geriatría y Gerontología, 43(3), 157-166. 